\title{
THE EFFECT OF CARBOXYMETHYL CHITOSAN/AMORPHOUS CALCIUM PHOSPHATE TO GUIDE TISSUE REMINERALIZATION OF DENTIN COLLAGEN
}

\author{
ROSDIANA NURUL ANNISA, NILAKESUMA DJAUHARIE, ENDANG SUPRASTIWI*, NORMA AVANTI
}

Department of Conservative Dentistry. Faculty of Dentistry. Universitas Indonesia, Jakarta, Indonesia. Email: esuprastiwi@yahoo.co.id

Received: 27 August 2018, Revised and Accepted: 07 February 2019

ABSTRACT

Objective: Carboxymethyl chitosan/amorphous calcium phosphate (CMC/ACP) can replace the role of dentine matrix protein 1. Guided tissue remineralization (GTR) is a method of extrafibrillar and intrafibrillar collagen remineralization. This study analyzed the ability of CMC/ACP to achieve intrafibrillar and extrafibrillar remineralization on demineralized dentin.

Methods: We divided 12 demineralized occlusal cavities into four groups: 7 days control group, 14 days control group, 7 days CMC/ACP group, and 14 days $\mathrm{CMC}$ /ACP group. In the control group, the cavities were directly filled with temporary restoration material, while the cavities of the CMC/ACP groups first underwent application of $\mathrm{CMC} / \mathrm{ACP}$ and were then filled with temporary restoration material. All samples were stored in a $37^{\circ} \mathrm{C}$ shaking incubator. 12 samples were analyzed by energy-dispersive X-ray (EDX) spectroscopy and four were analyzed by transmission electron microscope (TEM).

Results: From day 7 to day 14, there was a significant increase in calcium and phosphate levels within the CMC/APC groups. The phosphate levels were much lower than the calcium or minerals formed on the $7^{\text {th }}$ day, in the form of hydroxyapatite. TEM analysis shows that the CMC/ACP group exhibited more intrafibrillar and extrafibrillar remineralization.

Conclusion: CMC/ACP can improve the GTR process.

Keywords: Carboxymethyl chitosan/amorphous calcium phosphate, Guided tissue remineralization, Intrafibrillar remineralization.

(c) 2019 The Authors. Published by Innovare Academic Sciences Pvt Ltd. This is an open access article under the CC BY license (http://creativecommons. org/licenses/by/4. 0/) DOI: http://dx.doi.org/10.22159/ijap.2019.v11s1.16300

\section{INTRODUCTION}

Microscopically, the structure of dentin caries can be divided into two layers: Infected dentin and affected dentin. Infected dentin is the outermost layer of caries containing previously destroyed mineral matrix and apatite crystals, bacteria and their byproducts, decalcified tissues, and degraded collagen fibrils with disrupted collagen crosslinks. Meanwhile, on the affected dentin layer, denaturation has occurred, but it is still reversible and bacteria free [1,2].

Dentin collagens are composed of tropocollagen (triple helix/3 united polypeptide chains) with a length of $300 \mathrm{~nm}$, a diameter of $1.23 \mathrm{~nm}$, and fibrils with a wide gap of $67 \mathrm{~nm}$ at the end called the gap zone [3]. Based on the location of minerals in dentin collagen, there are intrafibrillar minerals found in the gap zone and extrafibrillar minerals found in the cavities that separate the collagen fibrils $[3,4]$.

Dentin remineralization can occur through two methods: Conventional remineralization/top-down process and guided tissue remineralization (GTR)/bottom-up process. Conventional/top-down remineralization occurs epitaxally, i.e., mineral deposition over the remaining apatite minerals. Meanwhile, in the GTR/bottom-up process, collagen remineralization occurs biomimetically using nanotechnology. Remineralization can be extrafibrillary or intrafibrillary. The GTR process begins with the formation of bonds between non-collagen protein analog material and amorphous calcium phosphate (ACP) nanoprecursors. This union forms nanocrystals that are able to enter the gap zone and then forms a larger apatite mineral structure [5].

The non-collagen protein in dentin is dentin matrix protein 1 (DMP1) which triggers the formation of ACP nanocluster precursors which then form the DMP1/ACP nanocomplex. DMP1 stabilizes ACP so that it remains nanosized and does not aggregate. The DMP1/ACP nanocomplex penetrates the gap zone and forms a hydroxyapatite crystalline for intrafibrillar remineralization [6].

Carboxymethyl chitosan (CMC) is a non-collagen protein analog material that is abundant in carboxyl and phosphate groups and stabilizes ACP nanoclusters to form CMC/ACP nanocomplexes (diameter $<40 \mathrm{~nm}$ ). This bond will penetrate into the gap zone for intrafibrillar remineralization $[2,6,7]$.

To analyze the occurrence of intrafibrillar remineralization, a device that can penetrate into the object of observation with image results is required. This study will analyze the potency of CMC/ACP on the extrafibrillar and intrafibrillar remineralization using transmission electron microscope (TEM) analysis. Afterward, the results can be confirmed by energy-dispersive X-ray (EDX) examination.

\section{METHODS}

This study was approved by the Ethics Committee of the University of Indonesia, Faculty of Dentistry (Ethics No. 133/Ethical Approval/ FKGUI/XII/2017-Protocol: 051301017).

CMC was obtained from the Research Laboratory of Sumatera Utara University. The manufacture of CMC/ACP preparations was according to the method described by Chen et al. [2]. We mixed $2.5 \mathrm{~g}$ of CMC into $40 \mathrm{ml}$ of water at a rotation speed of $1000 \mathrm{rpm}$ until dissolution. We then added by $0.498 \mathrm{~g}$ of $\mathrm{K}_{2} \mathrm{HPO}_{4}$ and the solution was mixed at $500 \mathrm{rpm}$.

After that, a solution comprising $0.555 \mathrm{~g}$ of $\mathrm{CaCl}_{2}$ mixed into $10 \mathrm{~mL}$ of deionized water was added dropwise into the CMC gel and stirred for 5 min until the formation of a CMC/ACP gel. The CMC/ACP gel was frozen at $-80^{\circ} \mathrm{C}$ for $2 \mathrm{~h}$ and lyophilized with a freeze-drying technique for $6 \mathrm{~h}$ to produce the final CMC/ACP preparation. 
The post-extracted premolar teeth were immediately immersed in a phosphate-buffered saline (PBS) solution and stored in a cooler at $4^{\circ} \mathrm{C}$ until the time of the experiment. We formed two cavities in each of 14 teeth using a diamond bur no. 16. Each of the 28 cavities was created with a depth of $3 \mathrm{~mm}$. We then divided the cavities into four groups, each group consisting of seven cavities. Group 1 was the 7 days control, Group 2 was the 14 days control, Group 3 was the 7 days CMC/ ACP group, and Group 4 was the 14 days CMC/ACP group. Before the treatment, all tooth surfaces with the exception of the cavities were coated with nail polish. All samples were then immersed in $17 \%$ EDTA (ethylenediaminetetraacetic acid) fluid for 1 week and stored in a shaking incubator at $37^{\circ} \mathrm{C}$. The tooth samples were rinsed with aquabidest for $30 \mathrm{~min}$ and immersed in $20 \mathrm{ml}$ of $1 \mathrm{M} \mathrm{NaCl}$ solution (pH 7.0) at $25^{\circ} \mathrm{C}$ for $8 \mathrm{~h}$. The purpose of immersing the samples in a $1 \mathrm{M}$ $\mathrm{NaCl}$ solution was to remove the easily soluble portion and keep the non-collagen protein present in dentin.

In the control group, the cavities were directly filled with composite resins while in the CMC/ACP group, before being filled with composite resins, the cavity bases were coated with CMC/ACP. Afterward, the teeth roots were immersed in a PBS solution and stored in a shaking incubator at $37^{\circ} \mathrm{C}$. Remineralization was observed on days 7 and 14 . Within each seven sample group, four samples were analyzed by EDX and three samples were analyzed by TEM.

Dentin samples were obtained by cutting the email and dentin portion down to the cavity base. We cleaned the samples with aquabidest and fixed them using the stratified dehydration method (i.e., immersion in ethanol concentrations of $50 \%, 70 \%, 80 \%$, and $90 \%$ for 20 min each, and finally, $96 \%$ for $2 \mathrm{~h}$ ). During the immersion in $96 \%$ alcohol for TEM examination purposes, the samples were scraped on the cavity base to remove the particles and directly soaked in $96 \%$ alcohol. The alcohol was allowed to evaporate until the sample was dry, and then, the particles were placed on top of a $3 \mathrm{~mm}$ diameter TEM grid. The samples were analyzed using a TEM. To analyze the calcium and phosphate levels following stratified dehydration, the samples were directly analyzed with EDX.

\section{RESULTS}

To examine the remineralization process, we measured calcium and phosphate levels using EDX. The data obtained were then analyzed subjected to one-way analysis of variance analysis, with a statistical significance level of $\mathrm{p} \leq 0.005$.

As seen in Table 1, both control and CMC/APC calcium and phosphate levels increased significantly from days 7 to 14 and calcium levels were significantly higher than the phosphate levels. When the four groups are compared, the results show that there is a significant difference between groups.

Comparative analysis of calcium levels (Table 2) revealed no significant difference between the control and CMC/ACP groups. Therefore, on days 7 and 14, both groups exhibited similar levels of releasing calcium.

There was a significant change in potassium levels within the control group $(p=0.001)$ and CMC/ACP group $(p=0.045)$ between days 7 and 14. Between-group comparisons of the control and CMC/ACP groups revealed no significant differences.

\section{DISCUSSION}

All samples were obtained from single-rooted premolar teeth that were extracted for orthodontic purposes to obtain uniform samples and immediately immersed in PBS solution and stored at $4^{\circ} \mathrm{C}$ to maintain tooth vitality.

Demineralized dentin represented affected dentine and was obtained by immersing the teeth in 17\% EDTA for 7 days stored in a shaker incubator at $37^{\circ} \mathrm{C}$. EDTA was chosen because it is a good chelating
Table 1: Mean, standard deviation, and significance value of the control and CMC/ACP groups (\%)

\begin{tabular}{llll}
\hline Group & \multicolumn{2}{l}{ Mean \pm SD } & p \\
\cline { 2 - 3 } & $\mathbf{7}$ days & $\mathbf{1 4}$ days & \\
\hline Calcium & & & $0.005^{*}$ \\
$\quad$ Control & $1.967 \pm 1.7995$ & $22.4500 \pm 0.57862$ & \\
$\quad$ CMC/ACP & $16.7567 \pm 7.6368$ & $35.456 \pm 13.3397$ & \\
Phosphate & & & \\
$\quad$ Control & $0.9297 \pm 0.695$ & $13.1700 \pm 1.504$ & \\
CMC/ACP & $7.3800 \pm 2.7458$ & $14.1333 \pm 3.366$ & \\
\hline * $<0.05$. CMC/ACP: Carboxymethyl chitosan/amorphous calcium phosphate
\end{tabular}

Table 2: Between-group comparisons of calcium levels

\begin{tabular}{|c|c|c|c|c|}
\hline \multirow[t]{2}{*}{ Group } & \multicolumn{2}{|c|}{ Control } & \multicolumn{2}{|c|}{ CMC/ACP } \\
\hline & 7 days & 14 days & 7 days & 14 days \\
\hline \multicolumn{5}{|l|}{ Control } \\
\hline 7 days & - & 0.071 & 0.285 & \\
\hline 14 days & & - & & 0.442 \\
\hline \multicolumn{5}{|l|}{$\mathrm{CMC} / \mathrm{ACP}$} \\
\hline 7 days & & & - & 0.109 \\
\hline
\end{tabular}

Significance value between the calcium groups $\mathrm{p} \leq 0.05$. CMC/ACP: Carboxymethyl chitosan/amorphous calcium phosphate

agent that can bind metal ions such as calcium. It was, therefore, able to remove all minerals in dentin while keeping the collagen intact $[8,9]$.

PBS solution was used as a medium for sample immersion because it contains sodium chloride, sodium phosphate, potassium chloride, and potassium phosphate which are ions present in body fluids. Thus, PBS can be a good source of calcium and phosphate ions for the process of remineralization [10]. Several studies have suggested that simulated body fluid (SBF) and PBS can be used as a remineralization medium $[2,8-10]$. The immersion medium selected in this study was PBS, in combination with a shaker incubator stored at $37^{\circ} \mathrm{C}$, to simulate the oral cavity environment [9]. Chen et al. used SBF as an immersion medium with peristaltic pumps that drained SBF into the root canal and pulp chamber [2].

GTR involves type 1 collagen, which acts as a scaffold, and apatite mineral. In addition, non-collagen proteins like DMP1 are also needed to bind with collagen and stabilize ACP to prevent aggregation and remain nanosized. The DMP1/ACP nanocomplex penetrates into the gap zones and forms octacalcium phosphate which transforms into a hydroxyapatite crystalline substance. In carious lesion, some DMP1 is damaged, so it needs a non-collagen protein analog material to replace DMP1 [6,11].

We tested CMC, a chitosan derivative with a carboxyl group that has good affinity with calcium ions for remineralization $[12,13]$. CMC stabilizes ACP to prevent its aggregation and allows it to remain nanosized and able to penetrate into the gap zone and form a hydroxyapatite crystalline during intrafibrillar remineralization [2].

As shown in Table 1, the levels of calcium and phosphate, both in the control and CMC/ACP groups, increased significantly from days 7 to 14 . This indicates that both the control and CMC/ACP group underwent remineralization. The occurrence of remineralization in the control group suggests that remineralization was naturally triggered by intact collagen within the demineralized dentin followed by the PBS immersion method and shaker incubator storage. The higher levels of calcium, compared to phosphate, both in the control and CMC/ ACP groups indicate that the bond formed on day 7 was already a hydroxyapatite bond. This finding agrees with Budiraharjo et al. [14] who found that remineralization commenced on day 3 with mineral penetration into the gap zones between the collagens in the form of 
Table 3: Between-group comparisons of phosphate levels

\begin{tabular}{llllll}
\hline Group & \multicolumn{2}{c}{ Control } & & & \multicolumn{2}{c}{ CMC/ACP } & \\
\cline { 2 - 3 } \cline { 5 - 6 } & $\mathbf{7}$ days & 14 days & & 7 days & 14 days \\
\hline Control & & & & \\
7 days & - & $0.001^{*}$ & & 0.056 & \\
$\begin{array}{l}\text { 14 days } \\
\text { CMC/ACP }\end{array}$ & & - & & 1.000 \\
7 days & & & & $0.045^{*}$ \\
\hline
\end{tabular}

*one-way ANOVA test with $\mathrm{p} \leq 0.05$. CMC/ACP: Carboxymethyl chitosan/ amorphous calcium phosphate

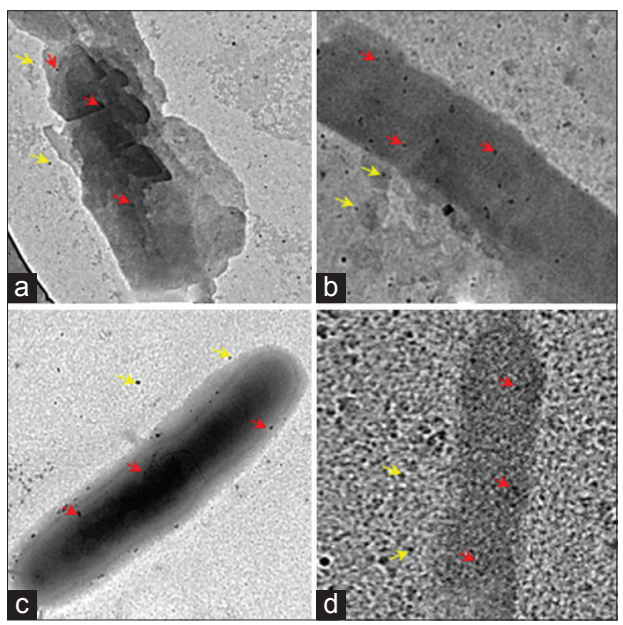

Fig. 1: Transmission electron microscope images of: (a) Control group day 7, (b) Control group day 14, (c) Carboxymethyl chitosan/amorphous calcium phosphate (CMC/ACP) group day 7 (d) CMC/ACP group day 14. The red arrows indicate black spots inside the collagen, suggestive of intrafibrillar remineralization. Yellow arrows show black spots outside the collagen, suggestive of extrafibrillar remineralization

octacalcium phosphate. Beginning on day 7, this substance transformed into hydroxyapatite crystals, achieving remineralization by day 14 [14]. Burwell et al. [15] also achieved intrafibrillar remineralization on affected dentine using a polyaspartic acid analog material beginning on day 7 and peaking on day 14 . Here, mineral deposits within the collagen gap zone were observed on day 7 , filling the collagen gap zone by day 14 [15].

Calcium and phosphate levels in the CMC/ACP group were significantly higher than the control group. This suggests that CMC/ACP improved remineralization because $\mathrm{CMC}$ is a non-collagen protein analog that is rich in carboxyl groups. These groups have a very high affinity to calcium and phosphate ions and are thus able to stabilize ACP nanoclusters. The resulting CMC/ACP nanocomplex penetrates the gap zone for intrafibrillar remineralization $[2,6,7]$.

The increased levels of calcium between the control and CMC/ACP group between days 7 and 14 were not statistically significant (Table 2), while the increased levels of phosphate between days 7 and 14 (Table 3), in both the control and CMC/ACP groups, were significantly different. This is consistent with the results of Budiraharjo et al. [14] and Burwell et al. [15], who observed hydroxyapatite minerals on day 7, thus resulting in much lower phosphate levels [14,15].

To observe the remineralization process, we use TEM imaging since this method can visualize nanosized particles and the mineral entry into the nanosized gap zone, signifying intrafibrillar remineralization [2]. TEM revealed both extrafibrillar and intrafibrillar remineralization. Extrafibrillar remineralization was indicated by minerals attached to the collagen surface, while intrafibrillar remineralization was indicated by the presence of mineral deposits in the gap zone.

Fig. 1 shows intrafibrillar and extrafibrillar remineralization (represented by black dots) in the control and CMC/ACP groups on days 7 and 14. The control group had fewer black dots than the CMC/ ACP group. On day 14, both the control and CMC/ACP groups had more mineral deposits in the gap zone than the control group on day 7 . This indicates intrafibrillar as well as extrafibrillar remineralization. However, when the control group was compared with CMC/ACP group at days 7 and 14, there was more intrafibrillar remineralization in the CMC/ACP on TEM evaluation. Several studies have reported that intrafibrillar mineralization may increase the mechanical properties of dentin, making it more resistant to demineralization while improving the elastic properties of collagen fibrils. Dentin collagen, with its high intrafibrillar mineral concentration, has a high elastic modulus $[4,16]$.

\section{CONCLUSION}

GTR occurred naturally in both the control and CMC/ACP groups. In the $\mathrm{CMC} / \mathrm{ACP}$ group, remineralization was better than in the control group. We, therefore, conclude that CMC/ACP improves extrafibrillar and intrafibrillar remineralization.

\section{CONFLICTS OF INTEREST}

There are no conflicts of interest to declare.

\section{REFERENCES}

1. Vaseenon S. Relationship between Caries-affected Dentin Mineral Density and Microtensile Bond Strength. MS Thesis, University of Iowa; 2011.

2. Chen Z, Cao S, Wang H, Li Y, Kishen A, Deng X, et al. Biomimetic remineralization of demineralized dentine using scaffold of $\mathrm{CMC} / \mathrm{ACP}$ nanocomplexes in an in vitro tooth model of deep caries. PLoS One 2015; 10: 0116553 .

3. Uskoković V, Bertassoni LE. Nanotechnology in dental sciences: Moving towards a finer way of doing dentistry. Materials (Basel) 2010;3:1674-91.

4. Bertassoni LE, Habelitz S, Kinney JH, Marshall SJ, Marshall GW Jr. Biomechanical perspective on the remineralization of dentin. Caries Res 2009:43:70-7.

5. Ferreira G, Ines M. Bioactive materials in dentin remineralization. Odontoestomatologia 2016;18:11-8.

6. Zhang X, Xiao Z, Wang H, Kishen A. Biomineralization and biomaterial considerations in dentin remineralization. JODE 2016;1:7-12.

7. Manuscript A, Nanostructures SPC. NIH Public Access. Nano 2008;6:2166-71.

8. Sousa SM, Silva TL. Demineralization effect of EDTA, EGTA, CDTA and citric acid on root dentin: A comparative study. Braz Oral Res 2005; $19: 188-92$

9. Toledano M, Osorio R. New advanced materials for high performance at the resin-dentine interface. Front Oral Biol 2015;17:39-48.

10. Uquillas JA, Kishore V, Akkus O. Effects of phosphate-buffered saline concentration and incubation time on the mechanical and structural properties of electrochemically aligned collagen threads. Biomed Mater 2011;6:035008.

11. Tjäderhane L, Carrilho MR, Breschi L, Tay FR, Pashley DH. Dentin basic structure and composition-an overview. Endod Top 2009;20:3-29.

12. Mourya VK, Inamdar NN, Tiwari A. Carboxymethyl chitosan and its applications. Adv Mater Lett 2010;1:11-33.

13. Farag RK, Mohamed RR. Synthesis and characterization of carboxymethyl chitosan nanogels for swelling studies and antimicrobial activity. Molecules 2012;18:190-203.

14. Budiraharjo R, Neoh KG, Kang ET, Kishen A. Bioactivity of novel carboxymethyl chitosan scaffold incorporating MTA in a tooth model. Int Endod J 2010;43:930-9.

15. Burwell AK, Thula-Mata T, Gower LB, Habelitz S, Kurylo M, Ho SP, et al. Functional remineralization of dentin lesions using polymerinduced liquid-precursor process. PLoS One 2012;7:e38852.

16. Dai L, Liu Y, Salameh Z, Khan S, Mao J, Pashley DH, et al. Can caries-affected dentin be completely remineralized by guided tissue remineralization? Dent Hypotheses 2011;2:74-82. 